\title{
Statistical and Stochastic Description of Microvoid Evolution Observed in Dynamic Failure of Ductile Materials*
}

\author{
Z.P. Huang ${ }^{1,2}$ and H.L. Li ${ }^{1}$ \\ ${ }^{1}$ State Key Laboratory of Nonlinear Mechanics, Institute of Mechanics, Chinese Academy of \\ Sciences, Beijing 100080, P.R. China \\ ${ }^{2}$ Department of Mechanics, Peking University, Beijing, 100871 P.R. China
}

Keywords: Microvoids, Statistical Evolution, Stochastic Fluctuation

\begin{abstract}
A brief review is presented of statistical approaches on microdamage evolution. An experimental study of statistical microdamage evolution in two ductile materials under dynamic loading is carried out. The observation indicates that there are large differences in size and distribution of microvoids between these two materials. With this phenomenon in mind, kinetic equations governing the nucleation and growth of microvoids in nonlinear rate-dependent materials are combined with the balance law of void number to establish statistical differential equations that describe the evolution of microvoids' number density. The theoretical solution provides a reasonable explanation of the experimentally observed phenomenon. The effects of stochastic fluctuation which is influenced by the inhomogeneous microscopic structure of materials are subsequently examined (i.e. stochastic growth model). Based on the stochastic differential equation, a Fokker-Planck equation which governs the evolution of the transition probability is derived. The analytical solution for the transition probability is then obtained and the effects of stochastic fluctuation is discussed. The statistical and stochastic analyses may provide effective approaches to reveal the physics of damage evolution and dynamic failure process in ductile materials.
\end{abstract}

\section{INTRODUCTION}

In order to reveal the nature of the dynamic failure process in materials, many attempts based on practical damage evolution have been made in recent years. It is shown that eventual failure of a damaged material may be caused by the nucleation, growth and coalescence of numerous microscopic voids or cracks. In fact, understanding of the mechanisms of dynamic failure, such as spallation under plate impact, is of scientific and technological interests. Different criteria for spalling were proposed in some early works on the dynamic failure problem, for example, the spall strength or critical tensile stress criterion (J.S. Rinehart, 1951, 1952), stress gradient criterion (Whiteman, 1962, Skidmore, 1965, Breed et al., 1968) and accumulative-damage criterion (Tuler and Butcher, 1968, Tuler, Davison, Stevens). These studies indicate that dynamic failure of materials is a process of damage evolution and the effects of damage evolution should be included in the analysis. Phenomenological descriptions were used in these early studies of continuum damage mechanics to deal with damage evolution in materials, and no detailed mechanisms for the initiation and propagation of microfractures were incorporated, partly because of the lack of the microscopic

- Supported by the National Natural Science Foundation of China (19632030) 
in situ experimental observation. A representative volume element containing voids or cracks was also considered to obtain the relationship between the macroscopic stress and the macroscopic strain in meso-damage mechanics.

The improvements in experimental techniques in more recent years provided powerful tools to reveal the physics based on which the evolution of microvoids and cracks proceeds prior to the occurrence of ultimate failure. In fact, the distribution of void or crack size in damaged materials can be evaluated by means of microscopic metallographic examination. In this respect, understanding the behavior of microdamage is only possible in terms of a statistical average sense, rather than from the individual void or crack. The damage variables, such as size, orientation and location, should be considered as certain kinds of statistical averages for the corresponding microscopic quantities. The microdamage states may be described by $q_{i}(i=1,2, \ldots, I)$ (e.g. the size of microcracks) and $p_{j}(j=1,2, \ldots, J)$ (e.g. the orientation and the location of microcracks). Then, the number density is defined by $n\left(q_{i}, p_{j}\right)$, i.e., the distribution of crack number versus crack size and/or orientation. The main purpose is to study the evolution law of $n\left(q_{i}, p_{j}\right)$, which should be determined according to the nucleation law and the growth law of microdefects.

The statistical study was carried out previously both theoretically and experimentally. Nucleation and growth model was proposed [1] to statistically describe the behavior of microscopic voids and/or cracks in damaged solids based on experimental observations in elaborate tests. The statistical investigation on the microscopic mechanisms provided a realistic basis for the development of computational models to simulate the whole failure process. Based on the balance law for crack number, an equation governing the statistical evolution of microcracks was proposed by Xing [2-4]. Complicated derivations were carried out based on the dislocation mechanisms for plastic deformation to find out the nucleation rate and growth rate of microcracks. The physics of strength and fracture toughness were examined. Bai et al. [5-6] investigated statistical evolution of ideal microcrack system in an impact experiment of aluminum alloy composites. A trans-scale (from meso- to macroscopic) analysis [17] was also carried out recently in an attempt to establish a quantitative/predictive approach for dynamic failure of materials based on the results of statistical mesoscopic damage mechanics. In these studies, Xing et al. gave more self-consistent descriptions or the damage evolution processes, and their attentions were mainly focused on the statistical chracteristics of microscopic cracks in brittle solids. However, the evolution of microvoids in ductile materials and its effect on macroscopic behavior of materials have not been systematically investigated yet taking into account the balance law for microvoid number. In fact, while low temperature or high strain-rate enhances brittle microcracking, high temperature or relatively low strain-rate favors ductile void nucleation and growth. During the failure of these materials caused by electric gun-driven plate impact or laser-driven shock wave, development of microvoids at different stages of evolution was observed (Sun et al. [9]). However, a series of fundamental questions largely remain unresolved. Thus, this topic is worthy of further investigation from the viewpoint of statistical treatment.

In the present work, experimental as well as theoretical analyses are carried out in an attempt to understand the statistical behavior of microvoid evolution in ductile materials under dynamic loading. Two different ductile materials with different strain-rate sensitivities-OFHC (copper) and L2 aluminum - are subjected to impact to obtain the statistical size distribution of microvoids. The experimental comparison indicates that there exist large differences in size and distribution of microvoids between these two materials. With this phenomenon in mind, the kinetic equations governing the nucleation and the growth of microvoids in nonlinear rate-dependent materials are combined with the balance law of void number to establish the statistical differential equations describing the evolution of microvoid number density. 


\section{EXPERIMENTAL OBSERVATION: MICROVOIDS' EVOLUTION UNDER DYNAMIC LOADING}

Electric gun-driven plate impact (EGDPI) technique was used to apply the uniaxial strain state in the specimen, which in turn caused microvoid evolution. Two different ductile materials with different levels of strain-rate sensitivity, OFHC copper and L2 aluminum, were tested.

\subsection{Electric Gun-Driven Plate Impact (EGDPI) Technique}

The schematic of EGDPI assembly is shown in Fig. 1. A pulsed current discharged from a capacitor made the metallic foil an exploding plasma of high temperature, pressure and density. Then, the exploding plasma drove a $0.05 \sim 0.1 \mathrm{~mm}$ thick Mylar flyer impacting the specimen at a velocity ranging $0.5 \sim 7 \mathrm{~km} / \mathrm{s}$. This impact velocity is far higher than that from elastic and plastic dynamic tests. Thus, instead of impacting the specimen directly, the Mylar flyer firstly hits the buffer. Then, a thicker metallic impactor (the secondary impactor) attached on the other surface of the buffer was driven and hit the target at a velocity of $10^{1} \sim 10^{2} \mathrm{~m} / \mathrm{s}$.

\subsection{Experimental Data and Observation}

The secondary impactors were designed to hit the target at different velocities by controlling the voltage of the capacitor in order to obtain different levels of damage. The impacted specimens were carefully sectioned and polished. The size and number of microvoids were measured on the cross-sectioned surface of specimens to obtain the surface distribution of void number versus void radius. Finally, the surface distributions were transformed to the volumetric distributions [15]. The statistical size distributions in the two materials with different levels of strain-rate sensitivity (OFHC copper and L2 aluminum)

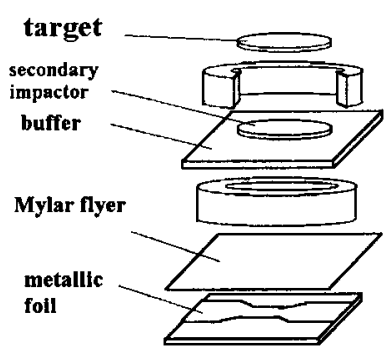

Fig. 1 EGDPI assembly subjected to the dynamic loading were then compared.

The target plates and the $\mathrm{L} 2$ aluminum secondary impactors were $2 \mathrm{~mm}$ and $0.5 \mathrm{~mm}$ in thickness, respectively. Thus, the specimens sustained a short stress pulse of about $0.2 \mu \mathrm{s}$ duration while successive nucleation, growth and coalescence of microvoids occurred in the specimens. The variation in impact velocity of secondary impact resulted in different amplitudes of stress pulse. As a result, different damage levels were obtained and investigated. The velocities of the secondary impactors were measured, the amplitude and time duration of the tensile pulse were estimated. Typical results of four specimens are listed in Table 1.

Table 1. Data of the Plate Impact Experiments

\begin{tabular}{ccccc}
\hline No. & $\begin{array}{c}\text { Materials of } \\
\text { Target }\end{array}$ & $\begin{array}{c}\text { Velocity of } \\
\text { Impactor(m/s) }\end{array}$ & Amplitude (GPa) & Time Duration $(\mu \mathrm{s})$ \\
\hline 1 & OFHC & 258 & 2.79 & 0.186 \\
2 & OFHC & 151 & 1.60 & 0.188 \\
3 & L2 Al & 253 & 1.98 & 0.177 \\
4 & L2 Al & 235 & 1.835 & 0.178 \\
\hline
\end{tabular}




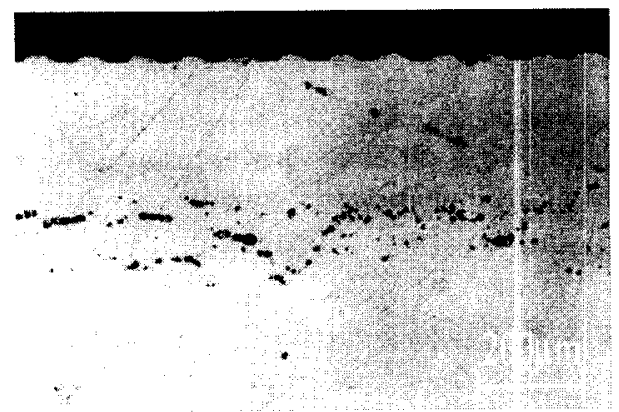

(a)

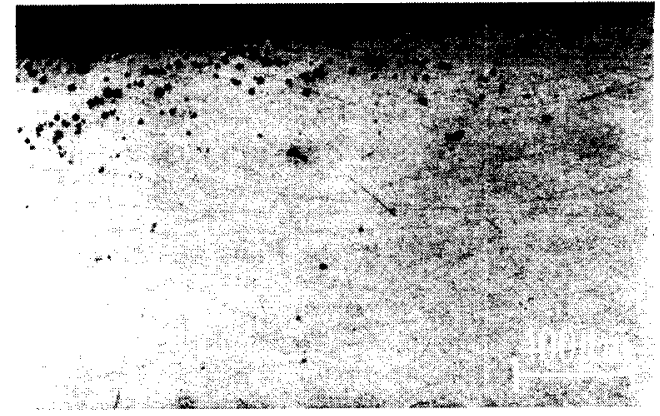

(b)

Fig. 2 Microvoids in specimen loaded by EGDPI ( (a)No.2, OFHC; (b)No.4, L2 Al)

Fig. 2 shows typical ductile void patterns on the cross-sectioned specimen surface. The cumulative size distribution of microvoids $\mathrm{N}(\mathrm{c})$, i.e., cumulative number of voids with size greater than $c$ per unit volume, were also measured. It is worth noting that, there exist large differences in size and distribution of microvoids between the two materials, that is, microvoids in OFHC (a ratesensitive material) were small and dense, whereas microvoids in L2 aluminum (a rate-insensitive material) appeared to be large but sparse. This phenomenon has not been theoretically explained before, nor have systematic comparisons between these materials with different rate sensitivities been reported.

The statistical analysis is presented in the next section to investigate the behavior of microvoids in ductile materials under dynamic loading, and thus to provide a theoretical explanation of the above phenomenon.

\section{THEORETICAL INVESTIGATION ON STATISTICAL BEHAVIOR OF MICROVOIDS}

\subsection{Basic Equations in the Statistical Description}

Based on the balance law of microvoid number, basic equations governing the evolution of size distribution of microvoids can be established. Together with the corresponding initial and boundary conditions, a set of equations can be written as

$$
\left.\begin{array}{l}
\frac{\partial n(c, t)}{\partial t}+\frac{\partial(\dot{c} n(c, t))}{\partial c}=0 \\
{[\dot{c} n(c, t)]_{c=b}=q(t)} \\
n(\infty, t)=0 \\
n(c, 0)=0 \quad(\text { where } c>b)
\end{array}\right\}
$$

where the number density of microvoids is denoted by $n(c, t)$, and the number of microvoids with sizes between $c$ and $c+\mathrm{d} c$ per unit volume at time $t$ is $n(c, t) \mathrm{d} c . b$ is a threshold size of microvoid (the size of nucleating void), $c$ is the radius of microvoid, $\dot{c}$ is the growth rate of microvoids, $f$ is the porosity, $q(t)$ is the nucleation rate and the number of new microvoids nucleated during $[t, t+\mathrm{d} t]$ per unit volume is $q(t) \mathrm{d} t$. 
The general solution for Eq. 1 can be expressed as

$$
n=\exp \left(\int_{c}^{b} \frac{1}{\dot{c}} \frac{\partial \dot{c}}{\partial c} \mathrm{~d} c\right) \cdot \Psi\left(t-\int_{b}^{c} \frac{\mathrm{d} c}{\dot{c}}\right),
$$

where the function $\Psi$ should be determined from the boundary condition Eq. 1 .

Without loss of generality, only the case of high stress triaxiality is considered. Suppose the macroscopic mean stress applied to a material element increases suddenly from zero to $\Sigma_{m}$ at time $t=0$, and its value is maintained constant thereafter. The evolution of microvoids in the material element is studied after such step loading. However, in order to solve the evolution equations, two kinetic equations for the nucleation and growth of microvoids should be supplemented.

\subsection{Nucleation and Growth Rate of Microvoids under High Stress Triaxiality}

As pointed out by Curran et al. [1], two types of microscopic nucleation mechanism are experimentally observed-tensile stress/temperature-driven nucleation and deformation-driven nucleation. In the case that temperature is high or the tensile mean stress is high compared with the deviatoric stress, the diffusion of vacancies into a cluster forms microscopic voids, thus the tensile stress/temperature-driven nucleation process predominates. This is the case the present study is mainly concerned. On the other hand, a relatively low triaxial stress or relatively high shear strain and near room temperature may favor the deformation-driven nucleation. The kinetics of nucleation processes is complex and is not fully understood so far. Hence, the expression of nucleation rate is usually given empirically. Seaman et al. [7] has suggested that the nucleation rate of several kinds of metals may be expressed by

$$
q=\dot{N}_{0} \cdot \exp \left[\frac{\Sigma_{m}-\Sigma_{n 0}}{\Sigma_{1}}\right],
$$

where $\Sigma_{m}$ is the macroscopic mean tensile stress and $\dot{N}_{0}, \Sigma_{n 0}, \Sigma_{1}$ are the material parameters. Eq. 3 indicates that the nucleation rate depends on the value of macroscopic stress under high stress triaxiality condition when the stress-driven nucleation process is predominant. Thus, for a constant temperature $T$ and a constant macroscopic stress $\Sigma_{m}$, the nucleation rate can be approximately taken as a constant $q$. Therefore, under a step loading, the nucleation rate can be expressed as

$$
q(t)=q \cdot H(t),
$$

where $H(t)=0$ if $t \leq 0$, and $H(t)=1$ if $t>0$.

The kinetic equation for the void growth rate may be obtained based on the analysis of mesodamage mechanics. An incompressible and power-law viscous matrix material is considered in the present study, its constitutive relation can be expressed as follows

$$
s_{i j}=\frac{2 \sigma_{0}}{3 \varepsilon_{e}}\left(\frac{\dot{\varepsilon}_{e}}{\dot{\varepsilon}_{0}}\right)^{m} \cdot \dot{\varepsilon}_{i j} \quad \text { or } \quad \dot{\varepsilon}_{i j}=\frac{3 \dot{\varepsilon}_{0}}{2 \sigma_{e}}\left(\frac{\sigma_{e}}{\sigma_{0}}\right)^{1 / m} \cdot s_{i j}{ }^{\prime}
$$

where $m(0 \leq m \leq 1)$ is the rate-sensitivity exponent of the matrix. $\dot{\varepsilon}_{e}=\sqrt{2 \dot{\varepsilon}_{i j} \dot{\varepsilon}_{i j} / 3}$ and $\sigma_{e}=\sqrt{3 s_{i j} s_{i j} / 2}$ are the effective rates of deformation and effective stress, respectively, $s_{i j}$ is the deviatoric stress, $\sigma_{0}, \dot{\varepsilon}_{0}$ are material constants. In the case of high stress triaxiality, the problem can approximately be reduced to a spherically symmetric one. Hence, by considering a hollow sphere as a unit cell, the void growth rate can be found [14]:

$$
\dot{c}=c \dot{\varepsilon}_{0} \cdot A\left(\Sigma_{m}, f\right),
$$


where

$$
A\left(\Sigma_{m}, f\right)=\frac{1}{2} \cdot\left[\frac{3 m}{2} \cdot \frac{\Sigma_{m} / \sigma_{0}}{1-f^{m}}\right]^{1 / m},
$$

and $f$ is the porosity defined by

$$
f=\frac{4 \pi}{3} \int_{0}^{\infty} c^{3} \cdot n(c, t) \mathrm{d} c=f_{0}+\int_{b}^{\infty} \frac{4 \pi}{3} \cdot c^{3} \cdot n(c, t) \mathrm{d} c,
$$

with its initial value being $f_{0}$.

From Eq. 4 and Eq. 6, solutions of Eq. 1 can be obtained and expressed in nondimensional form [14] with the nondimensional parameters defined by

$$
\bar{c}=c / b, \tau=\dot{\varepsilon}_{0} \cdot t, \quad \alpha=\frac{4 \pi q b^{3}}{3 \dot{\varepsilon}_{0}}, \quad \bar{n}(\bar{c}, \tau)=b \dot{\varepsilon}_{0} \cdot n(c, t) / q
$$

\subsection{Discussion based on the Theoretical Model}

The differences in the size distribution of microvoids in a rate-sensitive material and a relatively rate-insensitive one have been experimentally illustrated as mentioned in section 2 . In order to provide a theoretical explanation, several kinds of materials, which are rate-sensitive and relatively rate-insensitive (that is, with different $m$ values), with the same initial porosity $f_{0}$ are considered. Under the same step loading, the size distributions of microvoids for different $m$ are shown in Fig.3. For pure copper, a typical strain rate-sensitive material, the value of $m$ is large, and the peak of the distribution curve of number density $\bar{n}(\bar{c}, \tau)$ corresponds to relatively small size. The size corresponding to the peak becomes larger as $m$ decreases, while the whole curve for $\bar{n}(\bar{c}, \tau)$ becomes flat. On the other hand, for pure aluminum, a rate-insensitive material, $m$ is small and the radii of microvoids can reach relatively large values. Thus, from the above comparison, it can be concluded that, for rate-sensitive materials such as pure copper, the microvoids appear to be small and compact; for rate-insensitive materials such as pure aluminum, the microvoids appear to be big but sparse. This may explain the observed experimental phenomenon mentioned in section 2 . The evolution of the number density $\bar{n}(\bar{c}, \tau)$ is also shown in Fig. 4 .

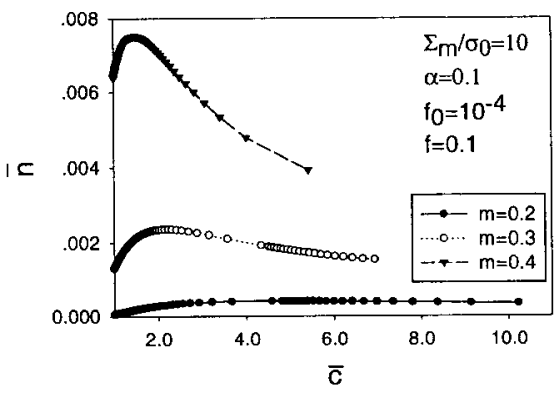

Fig. 3 Comparison of the number density curves for different value of $m$

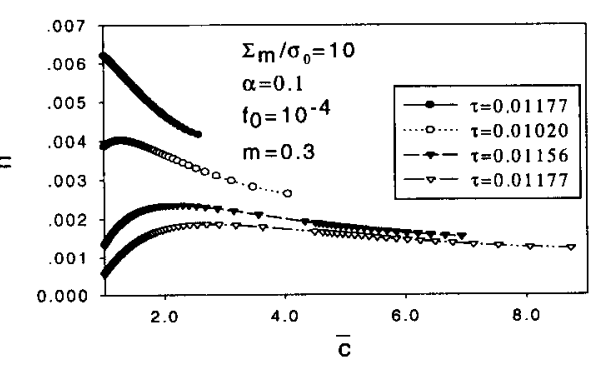

Fig. 4 Evolution of the number density curves 
Four nondimensional parameters, $m, \Sigma_{m} / \sigma_{0}, \alpha$ and the initial porosity $f_{0}$ are found to be governing parameters for the solution. The curve $\bar{n}(\bar{c}, \tau)$ is especially sensitive to the value of $m$, and the shape and order in magnitude of the curve will change if $m$ changes. It should be noted that, averaging the sample space gives the statistical property of the material. Thus, the statistical solution is a kind of mean-field solution, that is, the random deviation from the average value and its effect is not accounted for. Thus, this kind of model is deterministic, and will be referred to as the statistical model in the sequel.

\section{EFFECT OF STOCHASTIC FLUCTUATION: A STOCHASTIC GROWTH MODEL}

In this section, the effect of the random fluctuation will be considered. For each sample, the physical variable (growth rate, for example) may take a random value which deviates from the average one, or there exists a stochastic fluctuation in the average value. As a result, a probability should be introduced to describe the behavior of the random variables. In this way, a stochastic model may be defined in distinction from the statistical model mentioned above. For simplicity, only the stochastic fluctuation of void growth rate is included in the present analysis, which is called to as a stochastic growth model [10-12].

Based on the stochastic differential equation describing the growth rate of microvoids, a Fokker-Planck equation governing the evolution of the transition probability is derived, and the analytical solution for the transition probability is obtained. By taking the stochastic fluctuation into consideration, the evolution of microvoids number density is then discussed and compared with results of the deterministic statistical model. The validity of the statistical model and the stochastic analysis can be evaluated in this way.

\subsection{Stochastic Growth Model}

The inhomogeneous microscopic structure of materials may be regarded as an average structure background superimposed by an inhomogeneous fluctuation. The average structure is deterministic, whereas the inhomogeneous fluctuation is stochastic. Since the growth rate of microvoids depends closely on the microscopic structure of material, the fluctuation of microscopic structure may be regarded as many tiny disturbances, each of which changes the growth rate in a unpredictable but small way. Thus, the expression for the growth rate of microvoids may include a stochastic part which stems from the inhomogeneous fluctuation of microscopic structure, that is,

$$
\dot{c}=h(c, t)+\tilde{a}(c, t)
$$

where $c$ is the radius of microvoid. $h(c, t)$ is the deterministic part of growth rate, which is determined by the average background and the loading conditions, and is referred to as transport growth rate. Under the same step loading condition as described in section 3 , the transport growth rate can be obtained from the analysis of a hollow spherical unit cell. It is given by Eq. 6 , i.e.,

$$
h(c, t)=c \dot{\varepsilon}_{0} \cdot A\left(\Sigma_{m}, f(t)\right)
$$

$\tilde{a}(c, t)$ is the stochastic part of growth rate (fluctuation growth rate) determined by inhomogeneous fluctuation and local loading conditions. $\langle\widetilde{a}(c, t)\rangle=0$, where the bracket \langle\rangle indicates the ensemble average. In fact, the fluctuation growth rate $\tilde{a}(c, t)$ is assumed to have a Gaussian distribution with its mean value being zero and the correlation function being the Dirac delta function. Then, the stochastic differential equations (7) can be rewritten as a standard Langevin equation

$$
\dot{c}=h(c, t)+\sqrt{\frac{D}{2}} c \cdot \Gamma(t)
$$

where $D$ is the fluctuation growth coefficient, $\Gamma(t)$ is a stochastic term satisfying

$$
\langle\Gamma(t)\rangle=0, \quad\left\langle\Gamma(t) \Gamma\left(t^{\prime}\right)\right\rangle=2 \delta\left(t-t^{\prime}\right) .
$$


Thus, the fluctuation growth rate is expressed in terms of $c$ and $t$ with the level of fluctuation represented by the coefficient $D$ in Eq. (9).

A transition probability $P\left(c, t \mid c^{\prime}, t^{\prime}\right)$ should be introduced to describe the evolution process of microvoids in the present stochastic growth model. The transition probability is defined as a conditional probability density, or under the condition that the radius of voids at time $t^{\prime}\left(t^{\prime}<t\right)$ has the sharp value $c^{\prime}$, the probability to find the radius at time $t$ within the interval $[c, c+\mathrm{d} c]$ is $P\left(c, t \mid c^{\prime}, t^{\prime}\right) \mathrm{d} c$. Suppose the microvoid growth process can be viewed as a Markov process, the Fokker-Planck equation governing the evolution of transition probability can be derived from the Langevin equation (9) [13]

$$
\begin{aligned}
\frac{\partial P\left(c, t \mid c^{\prime}, t^{\prime}\right)}{\partial t}+\frac{\partial}{\partial c}\left\{\left[h(c, t)+\frac{D}{2} c\right] \cdot P\left(c, t \mid c^{\prime}, t^{\prime}\right)\right\} & \\
& -\frac{D}{2} \frac{\partial^{2}}{\partial c^{2}}\left[c^{2} \cdot P\left(c, t \mid c^{\prime}, t^{\prime}\right)\right]=0
\end{aligned}
$$

and the initial condition is $P\left(c, t^{\prime} \mid c^{\prime}, t^{\prime}\right)=\delta\left(c-c^{\prime}\right)$.

If the equations for $h(c, t)$ and $D$ are known, the Fokker-Planck equation (10) can then be solved to obtain the transition probability $P\left(c, t \mid c^{\prime}, t^{\prime}\right)$. The equivalent fluctuation growth coefficient $D$ will be evaluated later.

\subsection{Fluctuation Growth Rate of Microvoid}

The transport growth originates from the average microstructural background; whereas the fluctuation growth stems from the inhomogeneous fluctuation of microscopic structure of the material. Both of them depend on the microscopic structure of the same material, while they stem from different sides of the same object, hence they are related to each other. Therefore, the form of the stochastic part of growth rate may be obtained from the transport growth rate given above.

The transport growth rate (Eq. 8) is derived on the basis of an average microstructural background, so that the macroscopic stress $\Sigma_{m}$ and the porosity $f$ in Eq. 8 are ensemble average values. However, the stochastic fluctuation of the growth rate stems from the deviation of the local circumstance of each microvoid from the average condition due to the inhomogeneity of the material microstructure. Thus, the inhomogeneous fluctuation of material microstructures disturbs the local circumstance of each microvoid in an unpredictable but small way. As a result, microscopic stress field of the local circumstance may be assumed to take a random value which deviates from the average one for each microvoid. Therefore, the growth rate of each microvoid can be rewritten as

$$
\dot{c}=h(c, t)+\eta h(c, t)=h(c, t)+\eta \cdot \dot{\varepsilon}_{0} A\left(\Sigma_{m}, f\right) \cdot c \quad,
$$

and $\eta$ represents the relative effect of the deviation of the local circumstance. The first term at the right hand side of Eq. 11 is the average growth rate, and the second is the deviation from the average value caused by stochastic fluctuation of local conditions.

Then, we may evaluate the fluctuation growth coefficient $D$ which is related to the stochastic fluctuation factor $\eta$. Suppose the radius of a microvoid has the sharp value $c$ at time $t$, and after time $\Delta t$, the radius of this void is $c+\Delta c$, where $\Delta c$ is a random variable. From Eq. 7 :

$$
\begin{aligned}
& \Delta c=\int_{t}^{t+\Delta t} h(c, t) d t+\int_{t}^{t+\Delta t} \tilde{a}(c, t) d t=\langle\Delta c\rangle+\int_{t}^{t+\Delta t} \tilde{a}(c, t) d t \\
& \left\langle(\Delta c-\langle\Delta c\rangle)^{2}\right\rangle=\int_{t}^{t+\Delta t} d t_{1} \int_{t}^{t+\Delta t} d t_{2}\left\langle\tilde{a}\left(c, t_{1}\right) \cdot \tilde{a}\left(c, t_{2}\right)\right\rangle
\end{aligned}
$$


Eq. 12 can be expressed in terms of $D$. Substituting $\tilde{a}=\sqrt{D / 2} \cdot c \Gamma(t)$ into above equations, we arrive at

$$
\left\langle(\Delta c-\langle\Delta c\rangle)^{2}\right\rangle=\int_{t}^{t+\Delta t} d t_{1} \int_{t}^{t+\Delta t} d t_{2} D c^{2} \cdot\left\langle\Gamma\left(t_{1}\right) \cdot \Gamma\left(t_{2}\right)\right\rangle / 2 \cong D c^{2} \cdot \Delta t
$$

where $\Delta c / c$ is supposed to be a small quantity, and only small quantities of the lowest order are remained.

On the other hand, Eq. 12 can also be expressed in terms of $\eta$ based on Eq. 11. By ignoring the small change of the term $\dot{\varepsilon}_{0} A\left(\Sigma_{m}, f\right)$ during the time interval $[t, t+\Delta t]$,

$$
\int_{t}^{t+\Delta t} \dot{\varepsilon}_{0} A\left(\Sigma_{m}, f\right) \mathrm{d} t=\dot{\varepsilon}_{0} A\left(\Sigma_{m}, f\right) \cdot \Delta t
$$

The integration of Eq. 11 gives

$$
c+\Delta c=c \cdot \exp \left[\dot{\varepsilon}_{0} A\left(\Sigma_{m}, f\right)(1+\eta) \Delta t\right]=\langle c+\Delta c\rangle \cdot \exp \left[\eta \dot{\varepsilon}_{0} A\left(\Sigma_{m}, f\right) \Delta t\right]
$$

Therefore, we have

$$
\left\langle(\Delta c-\langle\Delta c\rangle)^{2}\right\rangle=\left\langle(c+\Delta c-\langle c+\Delta c\rangle)^{2}\right\rangle=\left\langle(c+\Delta c)^{2}\right\rangle-\langle c+\Delta c\rangle^{2} \cong c^{2} \cdot 2\left\langle\eta^{2}\right\rangle\left[\dot{\varepsilon}_{0} A\left(\Sigma_{m}, f\right) \Delta t\right]^{2}
$$

Comparing Eqs. 13 and 14, we obtain

$$
D=2\left\langle\eta^{2}\right\rangle \dot{\varepsilon}_{0}^{2} A^{2}\left(\Sigma_{m}, f\right) \Delta t=2 \beta^{2} \dot{\varepsilon}_{0}^{2} A^{2}\left(\Sigma_{m}, f\right) \Delta t
$$

where $\beta=\sqrt{\left\langle\eta^{2}\right\rangle}=\frac{\sqrt{\left\langle\tilde{a}^{2}\right\rangle}}{h(c, t)}$ is the relative intensity of the stochastic fluctuation of the growth rate.

\subsection{Transition Probability and Microvoid Number Density}

In view of Eqs. 8 and 15 (here $\Delta t=t-t^{\prime}$ ), the differential equation (Eq. 10) can be solved, and the transition probability may be expressed in a closed form as

$$
P\left(c, t \mid c^{\prime}, t^{\prime}\right)=\frac{\exp \left\{-\frac{\left[\ln \left(c / c^{\prime}\right)-\dot{\varepsilon}_{0} A\left(t-t^{\prime}\right)\right]^{2}}{D\left(t-t^{\prime}\right)}\right\}}{\eta_{1} \sqrt{\pi} \sqrt{D\left(t-t^{\prime}\right)} \cdot c} \cdot H\left(c-c^{\prime}\right)
$$

where $H(c)$ is the Heaviside step function, $\eta_{1}$ is a normalization factor determined by the normalization condition $\int_{0}^{\infty} P\left(c, t \mid c^{\prime}, t^{\prime}\right) \mathrm{d} c=1$. If $\beta$ is relatively small, then $\eta_{1} \cong 1$.

The stochastic evolution process of microvoids may be regarded as a Markov process, hence the probability density function at time $t$ is completely determined by the probability distribution function at time $t^{\prime}\left(t^{\prime}<t\right)$, and is independent of the probability density function at all earlier time. Thus, the evolution of $n(c, t)$ can be expressed as

$$
n(c, t)=\Delta n_{N}+\int_{b}^{c} n\left(c^{\prime}, t^{\prime}\right) P\left(c, t \mid c^{\prime}, t^{\prime}\right) \mathrm{d} c^{\prime}
$$

where $\Delta n_{N}$ represents the contribution of the nucleation process during the time interval $\left[t^{\prime}, t\right]$.

Under the same step loading conditions described in section 3, the nucleation term $\Delta n_{N}$ in Eq.(17) can be rewritten as follows according to the analysis of the deterministic growth model[14]

$$
\Delta n_{N}=\frac{q}{b \dot{\varepsilon}_{0}} \frac{1}{A c} H\left(\Delta t-\frac{\ln (c / b)}{A \dot{\varepsilon}_{0}}\right)
$$


where $\Delta t=t-t^{\prime}$. Finally, the transition probability can be rewritten in nondimensional form by defining $\bar{c}^{\prime}=c^{\prime} / b, \bar{c}=c / b, \tau^{\prime}=\dot{\varepsilon}_{0} t^{\prime}, \tau=\dot{\varepsilon}_{0} t, \Delta \tau=\dot{\varepsilon}_{0} \Delta t$ and $\bar{P}\left(\bar{c}, \tau \mid \bar{c}^{\prime}, \tau^{\prime}\right)=b \cdot P\left(c, t \mid c^{\prime}, t^{\prime}\right)$.

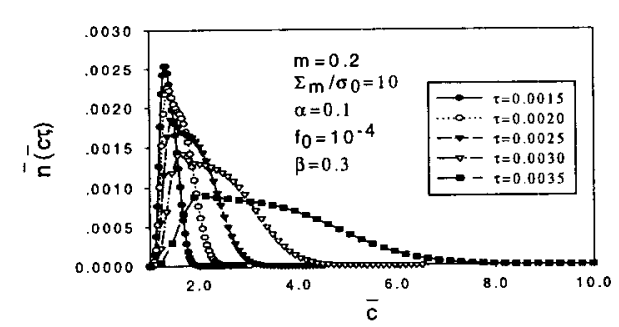

(a)

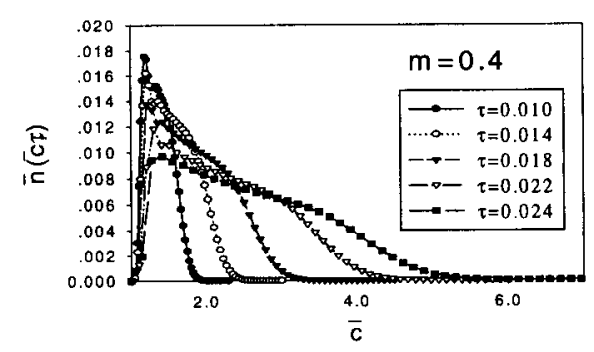

(b)

Fig. 5 The evolution of the number density $\bar{n}(\bar{c}, \tau)$

\subsection{Numerical Results and Discussion}

The curves of number density $\bar{n}(\bar{c}, \tau)$ vary with time and the evolution processes are shown in Fig.5. The strain rate sensitive exponent $m$ takes different values $(m=0.2,0.4)$ in Fig $5(\mathrm{a}, \mathrm{b})$. Thus, the effects of strain rate-sensitivity may be illustrated when we compare the curves in these figures. In Fig. $5 \mathrm{a}(m=0.2$, a rate-insensitive material like pure aluminum), for a rather short duration ( $\tau=0.0035)$, the peak of the curve $\bar{n}(\bar{c}, \tau)$ corresponds to $\bar{c}=2.0$; whereas in Fig. $5 \mathrm{~b}$ ( $m=0.4$, a rate-sensitive material like pure copper), for a rather longer time duration $(\tau=0.024)$, the peak of the curve $\bar{n}(\bar{c}, \tau)$ corresponds to a value still less than 2.0 and the peak of curve $\bar{n}(\bar{c}, \tau)$ moves toward the direction of larger void size much more slowly than that for rate-insensitive ones. All these curves become flatter as time increases. However, it proceeds much more rapidly for the rateinsensitive materials. Again, we can conclude (based on the curves obtained from the present stochastic model) that, for rate-sensitive materials such as pure copper, the microvoids appear to be small and compact; for rate-insensitive materials such as pure aluminum, the microvoids appear to be big but sparse. This is consistent with the experimental observation mentioned in section 2 .

In Fig. 6 , the number density curve $\bar{n}(\bar{c}, \tau)$ obtained from the stochastic growth model is compared with that from the statistical (deterministic) model. The parameter $\beta$ represents the relative intensity of the stochastic fluctuation of the void growth rate. $\beta=0$ corresponds to the result of the deterministic growth model. It can be seen from Fig. 6 that as $\beta$ approaches zero, the stochastic model can be reduced to the statistical (deterministic) one. This confirms the validity of the present stochastic analyses. When $\beta$ takes a rather larger value, the effect of the stochastic fluctuation can be examined in detail. It should be mentioned that, the present

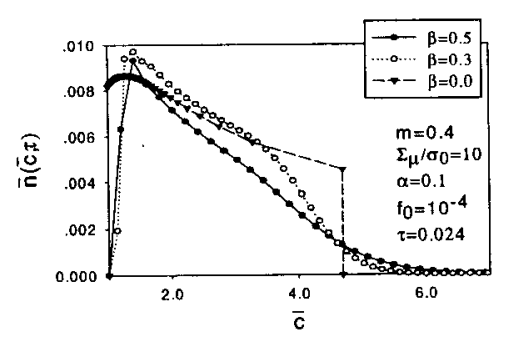

Fig. 6 Comparison between the result of the stochastic growth model and the deterministic result stochastic model works well when the state is not far away from the mean field. When the mean-field approximation holds, the relative fluctuation can be viewed as small quantities in our derivation to obtain the transition probability $P\left(c, t \mid c^{\prime}, t^{\prime}\right)$. 
However, as the damage evolution proceeds, the deviation of the local condition for each microvoid from the average one may become stronger. This means that the samples with slight initial mesoscopic differences may exhibit significant different failure behavior. The fluctuations become a predominate feature as samples are loading to eventual failure. Averaging over sample space becomes meaningless for such a process far from equilibrium. Thus, the present stochastic analysis is no longer valid when the failure of materials are about to occur. Owing to the complexity during this stage, analytical description may become extremely difficult, and one may have to resort to numerical simulation to reveal characteristics of such states far from equilibrium. From the simulation of random fuse networks [16], it was shown that it is the dynamic evolution which leads to the strong amplification of differences between the samples as they are about to fail. Meanwhile, there is also a significant deviation from the homogeneous distribution. Damage distribution in the specimen becomes localized and the stochastic fluctuations in this damage localized regime become extremely strong. As a common phenomenon in failure model of disorded media, the socalled 'evolution induced catastrophe' [16] can be often observed. Nevertheless, the early period of damage accumulation (before the transition from a state not far away from a mean-field to a catastrophic state) possesses a large portion of the whole life of samples, and the statistical model as well as the stochastic analysis should be valid during the most of time when the samples sustain relatively severe damage. Thus, the statistical and stochastic treatment may reveal some features of the damage accumulation stage not far away from the mean-field, which is important for understanding of the initiation of damage localization and the complete failure processes. In fact, a recent investigation [17] indicates that a trans-scale analysis based on the statistical investigation results in an effective prediction of the occurrence of damage localization, which may serve as a possible precursor to dynamic failure.

\section{CONCLUSIONS}

A comparative study of statistical microdamage evolution in two ductile materials is carried out under dynamic loading. Experimental observation indicates that, for rate-sensitive materials like OFHC copper, microvoids in the materials are smaller but dense, whereas for rate-insensitive materials like L2 aluminum, microvoids are larger but sparse. The kinetic equations governing the nucleation and the growth of microvoids in ductile materials are combined with the balance law of void number to obtain the evolution history of size distributions of microvoids. The theoretical solution may provide a reasonable explanation for the experimental phenomena mentioned above. A stochastic growth model is considered to include the effects of stochastic fluctuation originating from the inhomogeneous microstructures. The analytical solution for the transition probability is then obtained. The size distribution curves obtained from the stochastic growth model and that from the statistical (deterministic) model agree to each other when the relative intensity of stochastic fluctuation of growth rate is small. On the whole, the statistical and stochastic analyse may provide an effective approach to reveal the physics of damage evolution and dynamic failure of materials.

\section{REFERENCES}

1. D.R. Curran, L. Seaman, D.A. Shockey, Phys. Rep., 147 (1987), pp. 253-388.

2. X.S.Xing, ACTA Physica Sinica, 22 (1966), pp. $487-497$.

3. X.S. Xing, Eng. Frac. Mech., 26 (1987), pp. 393-419.

4. X.S.Xing, Eng. Frac. Mech., 37 (1990), pp. 1099-1104.

5. F.J. Ke, Y.L Bai , M.F.Xia, Science in China, Series A (1990), pp. 621-631.

6. Y.L. Bai , Z. Ling, L.M. Luo, F.J. Ke, J. Appl. Mech., 59 (1992), pp. 622-627. 
7. L.Seaman, D.R.Curran, D.A.Shockey, J. Appl. Phys., 47 (1976), pp. 4814-4826.

8. L.Z. Sun, Z.P.Huang, Int. J. plasticity, 8 (1992), pp. 903-924.

9. C.W.Sun, S.P.Feng. X.P.Long, X.Yuan, Z.P.Huang, J. De Physique IV Colloque C8, 4 (1994), pp. 355-360.

10. J.L. Bogdanoff, F. Kozin, Probabilistic Models of Cumulative Damage, John Wiley \& Sons, NewYork (1985).

11. X.S.Xing, Eng. Fract. Mech., 24 (1986), pp. 45-64.

12. M.F. Xia , F.J. Ke, Y.L. Bai, Science in China, Series A (in Chinese), (1991), p. 276.

13. H. Risken, The Forkker-Planck Equations, Springer-verg, (1984).

14. H.L.Li, Z.P.Huang, Science in China, Series A, 39 (1996), pp. 1332-1339.

15. L. Seaman, D.R.Crewdson, J. Appl. Phys., 49 (1978), pp. 5221.

16. M. F. Xia, Z. Q. Song, J.B. Xu, K.H. Zhao, Y.L. Bai, Commun. Theor. Phys., 25 (1996), pp. 4954.

17. Y.L.Bai, M.F.Xia, F.J.Ke and H.L.Li, in Proc. IUTAM Symp. Rheology of Bodies with Defects, R.Wang, ed., Kluwer Academic Publisher (1998), pp. 55-66. 
Advances in Engineering Plasticity

10.4028/www.scientific.net/KEM.177-180

Statistical and Stochastic Description of Microvoid Evolution Observed in Dynamic Failure of Ductile Materials

10.4028/www.scientific.net/KEM.177-180.1

\section{DOI References}

[3] X.S. Xing, Eng. Frac. Mech., 26 (1987), pp. 393-419.

doi:10.1016/0013-7944(87)90021-X

[8] L.Z. Sun, Z.P.Huang, mt. J. plasticity, 8 (1992), pp. 903-924.

doi:10.1016/0749-6419(92)90042-B 